\title{
Role of erlotinib in the management of pancreatic cancer
}

\author{
Naureen Starling' \\ John Neoptolemos ${ }^{2}$ \\ David Cunningham ${ }^{3}$
}

'Royal Marsden Hospital, Sutton, Surrey, UK; ${ }^{2}$ School of Cancer Studies, University of Liverpool, Royal Liverpool University Hospital, Liverpool, UK; ${ }^{3}$ Gastrointestinal and Lymphoma Units, Royal Marsden Hospital, Sutton, Surrey, UK
Correspondence: John Neoptolemos Division of Surgery and Oncology, Royal Liverpool University Hospital, Daulby Street, Liverpool L69 3GA, UK

Tel +44 I5I 7064 I75

Fax +44 I5I 7065798

Email j.p.neoptolemos@liv.ac.uk

\begin{abstract}
Pancreatic cancer is a largely chemo-resistant disease with a poor prognosis. Despite the adoption of gemcitabine monotherapy as a standard of care, outcomes remain poor. Until recently randomized phase III studies have not demonstrated superiority of various cytotoxic combinations or a number of the newer biologic targeted drugs. The situation has changed with capecitabine and erlotinib, either of which in combination with gemcitabine produces a small increase in survival. Erlotinib is a small molecule tyrosine kinase inhibitor against epidermal growth factor receptor which has an important role in the molecular pathogenesis of pancreatic cancer. In both pre-clinical and early clinical evaluation it has shown anti-tumor activity against pancreatic cancer in combination with gemcitabine. A randomized phase III study in locally advanced and metastatic pancreatic cancer has shown a survival advantage for the combination of gemcitabine plus erlotinib over gemcitabine alone. The rationale for the clinical development of erlotinib in combination with gemcitabine in pancreatic cancer culminating in this randomized trial, together with pharmacologic, toxicity and patient selection considerations form the focus of this review.
\end{abstract}

Keywords: erlotinib, tarceva, capecitabine, xeloda, gemcitabine, epidermal growth factor receptor, pancreas, pancreatic

\section{Introduction}

Pancreatic cancer is an aggressive malignancy, the vast majority of patients presenting with advanced unresectable disease. Despite advances in the development of conventional chemotherapy, notably the establishment of gemcitabine as a standard of care, response rates to therapy are low and survival from the disease is still depressingly poor. Gemcitabine gained favor in the treatment of advanced pancreatic cancer worldwide after Burris et al (1997) reported the results of their randomized controlled trial comparing gemcitabine, a novel nucleoside analogue with bolus 5fluorouracil (5-FU). This demonstrated significantly improved clinical benefit response rates $(24 \%$ vs $5 \% ; \mathrm{p}=0.0022)$ and median survival duration (5.65 vs 4.4 months; $p=0.0025$ ) for the gemcitabine arm. The one-year survival rate was $19 \%$ in the gemcitabine group and only $2 \%$ in the 5 -FU treatment group. The response rate was $5.4 \%$ versus $0 \%$ (nonsignificant) and stable disease $39 \%$ versus $19 \%$ in favour of gemcitabine. Despite the modest but significant increment in survival, gemcitabine was adopted as the standard of care based on the significant improvement in clinical benefit response which is a composite measure of pain (intensity and analgesic requirement), performance status and weight. There are data suggesting that modulating the rate of infusion of gemcitabine may enhance anti-tumor activity with a randomized phase II study of fixed-dose rate gemcitabine given at an infusion rate of $10 \mathrm{mg} / \mathrm{m}^{2} / \mathrm{min}$, demonstrating a trend towards improvement in response rate and survival when compared with standard infusion of gemcitabine over thirty minutes (Tempero et al 2003).

Gemcitabine has become the reference arm in randomized trials in the treatment of advanced pancreatic cancer to which newer agents have been tested against either 
alone or in combination, particularly doublets. However, several alternative cytotoxics as part of single agent or combination therapies (usually doublets) have failed to produce superior results over gemcitabine alone (Cheverton et al 2004; Richards et al 2004; Rocha Lima et al 2004; Louvet et al 2005). Recently a UK randomized phase III study of 533 patients with advanced pancreatic cancer reported significantly improved survival for the combination of gemcitabine plus capecitabine over gemcitabine alone with a median overall survival of 6.0 months versus 7.4 months in favor of the combination arm (hazard ratio [HR] $0.80 ; 95 \%$ confidence interval [CI]: $0.65,0.98: \mathrm{p}=0.026$ ) and 12 month survival of $19 \%$ and $26 \%$ respectively (Cunningham et al 2005). Combination therapy was well tolerated. This represents the first positive phase III study demonstrating superiority of a cytotoxic doublet over gemcitabine monotherapy. These results contrast with a recently reported negative Swiss phase III study of gemcitabine and capecitabine in which the doublet was administered according to a different dosing and schedule to that used in GEMCAP (Herrmann et al 2005).

There is still a clear need for new therapies and the identification of novel therapeutic targets in an attempt to improve on current standards. Within the last decade there have been significant advances in our understanding of the molecular pathogenesis underlying the development and progression of pancreatic cancer. A greater understanding of the interplay between tumor, stroma, and host and of important genetic and epigenetic events has been vital in identifying and developing potential therapeutic interventions with the capacity to disrupt tumor progression. A few randomized studies of gemcitabine versus gemcitabine plus a biologic agent directed at promising novel targets have proved negative (Bramhall et al 2001, 2002; Moore et al 2003; Van Cutsem et al 2004). The reasons for this are unclear and for some of the candidate targets may reflect that in metastatic pancreatic cancer, and indeed many cancers, cell proliferation is likely to be dependent on more than one genetic lesion such that growth control is non-linear. The biologics have often been most successful when targeting a critical genetic lesion upon which cellular proliferation has become dependent, the notion of oncogene addiction.

The contribution of the epidermal growth factor receptor (EGFR) pathway to oncogenesis has been well documented and therapeutic exploitation of this axis has proved to be a successful strategy in several other tumor types including colorectal and head and neck cancers. There has been considerable interest in targeting the EGFR pathway in advanced pancreatic cancer with agents such as erlotinib currently in the limelight. The application of erlotinib to the treatment of pancreatic cancer will be the focus of this review.

\section{Targeting the epidermal growth factor pathway in pancreatic cancer}

The erbB family of growth tyrosine kinase receptors comprises four structurally homologous members including erbB1, also known as the epidermal growth factor receptor (EGFR), erbB2 (HER2/neu), erbB3 (HER3) and erbB4 (HER4) (Yarden and Sliwkowski 2001). ErbB tyrosine kinase receptors are functionally inactive monomers that contain an extracellular ligand binding domain, a single hydrophobic transmembrane domain and an intracellular tyrosine kinase domain. Binding of one of over ten cognate ligands results in receptor homo- or hetero-dimerisation mediated by cysteine rich loops in the ectodomain (Ogiso et al 2002). The resulting phosphorylation of tyrosine residues within the adenosine triphosphate (ATP) binding kinase domain activates kinase activity and results in phosphorylation of residues in the regulatory carboxyl terminal tail of the receptor. This leads to the phosphorylation, activation and recruitment of signaling effectors containing SRC homology 2 and phosphotyrosine binding domains that initiate a cascade of downstream signaling events ultimately culminating in gene transcription (Marmor et al 2004).

This complex network of erbB receptors, their associated ligands, and the various signal processing pathways contributes to the intricate regulation of normal cellular processes including proliferation, differentiation, cell motility, and survival. However, dysregulation of the network can lead to aberrant control of cell growth which may potentiate malignant transformation. Of the various components of the network, abnormal signaling through the erbB1 and erbB2 receptors has been most widely studied and implicated in the pathogenesis of several tumor types including pancreatic cancer. The EGFR, in particular, has been selected as a rational target in the treatment of this disease.

\section{EGFR signaling in pancreatic cancer}

Aberrant signal transduction through EGFR and HER2 influences several processes pertinent to cancer progression 
including proliferation, resistance to apoptosis, invasion, angiogenesis, and metastasis. Dysregulation of the EGFR signaling pathway may occur through various mechanisms including receptor or ligand overexpression, receptor mutation (as with EGFRvIII which has a truncated extracellular domain and demonstrates constitutive ligandindependent activation) and receptor crosstalk (Arteaga 2003). In contrast to receptor homodimerization, EGFR heterodimerization, for instance with HER2, provides a stronger mitogenic stimulus mediated predominantly through the ras-raf mitogen activated protein kinase (MAPK) and AKT-PI3-kinase (phosphatidylinositol 3kinase) pathways (Figure 1) (Mendelsohn and Baselga 2003; Marmor et al 2004). The latter leads to degradation of the inhibitory IKK protein and translocation of the nuclear factor $\kappa \mathrm{B}(\mathrm{NF}-\kappa \mathrm{B})$ into the nucleus where it activates transcription of genes involved in cell survival and chemoresistance (Guttridge et al 1999; Hinz et al 1999). The epidermal growth factor has been shown to activate NF- $\mathrm{KB}$ in smooth muscle cells, A431 cells, fibroblasts and estrogen receptor negative, EGF-overexpressing breast cancer cell lines (Habib et al 2001). HER2 has no cognate ligand but acts as a dimerization partner for other members of the erbB family utilizing the same effector signaling pathways and strengthening the mitogenic stimulus through such means as decreasing the rate of receptor downregulation and more efficient coupling to signaling pathways (Marmor et al 2004).

Molecular dysfunction of the EGFR signal transduction axis in pancreatic cancer can occur at several levels. A significant proportion of pancreatic cancers demonstrate increased expression of EGFR and its cognate ligand and

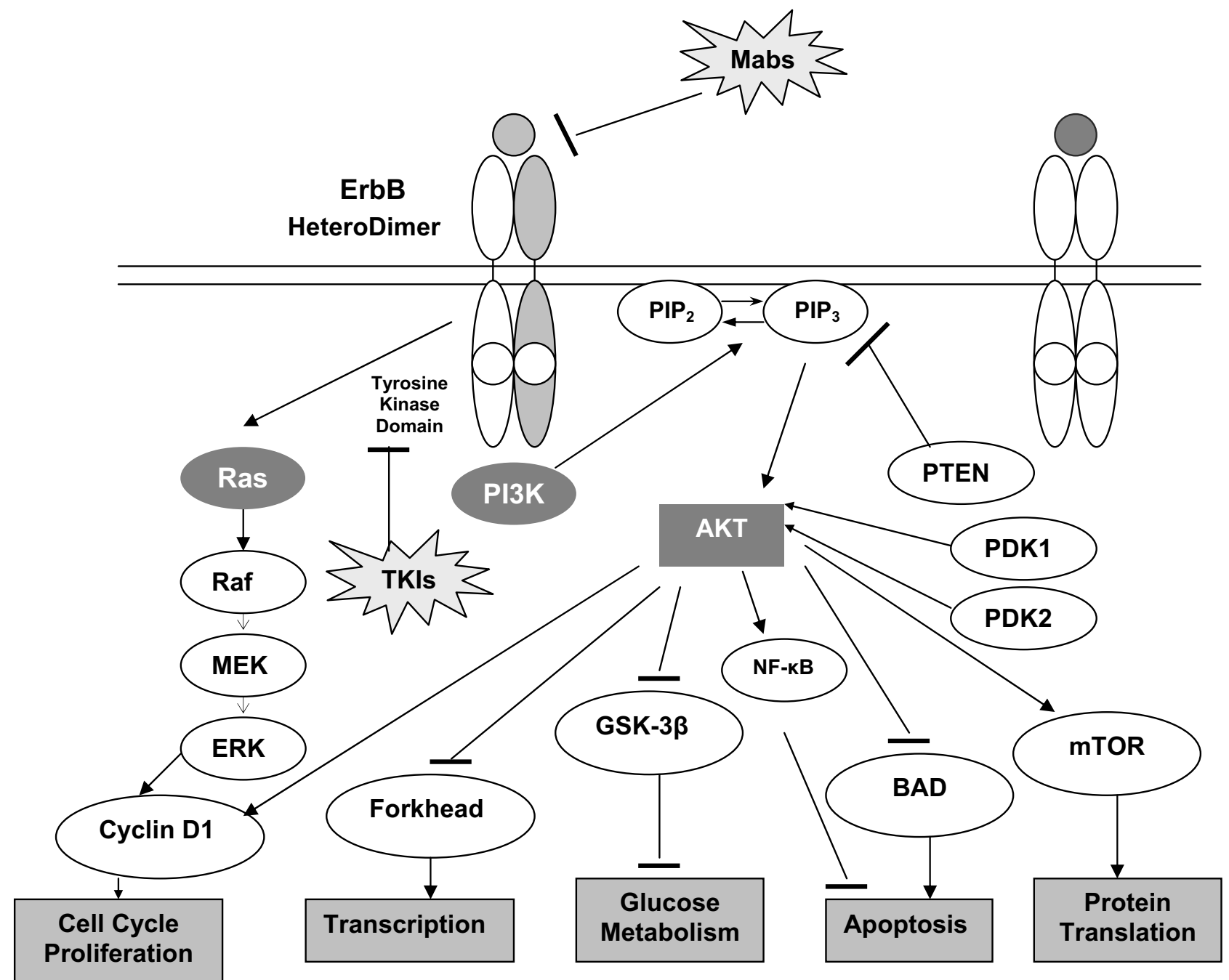

Figure I The epidermal growth factor receptor pathway. Signaling through the epidermal growth factor receptor initiates a cascade intracellular cell signaling events which result in proliferation, angiogenesis and cell survival.

Abbreviations: BAD, pro-apoptotic protein of the bcl-2 family; ERK: extracellular signal regulated kinase; GSK: glycogen synthase kinase; Mabs, monoclonal antibodies; MEK: mitogen-activated protein kinase; mTOR, mammalian target of rapamycin; NF-kB, nuclear factor-kappa B; PDK: 3-phosphoinositide dependent kinase; PI3K, phopsphatidylinositol-3-kinase; PIP3: phosphatiyidylinositol (3,4,5)-triphosphate; PTEN, phosphatase and tensin homolog detected on chromosome I0; Ras/Raf: Serine/threonine kinase family; TKIs, tyrosine kinase inhibitors. 
this has been correlated with enhanced tumor aggressiveness and a worse prognosis (Lemoine et al 1992; Yamanaka et al 1993). Akt-2, a molecule downstream of PI3 kinase in the EGFR signal cascade is overexpressed in up to $60 \%$ of human pancreatic biopsies and the Akt-2 oncogene is amplified in 10\%-20\% of pancreatic cancer cells (Ruggeri et al 1998; Schlieman et al 2003). Akt has demonstrated basal phosphorylation and activation in pancreatic cell lines which may confer resistance to apoptosis (Bondar et al 2002; Schlieman et al 2003). Akt activation may in some cases result from loss of the inhibitory effect of PTEN and whilst PTEN is not known to be mutated in pancreatic cancer, loss of function could occur through alternative mechanisms (Perren et al 2000; Ebert et al 2002). Further downstream in the signaling cascade constitutive activation of the transcription factor NF- $\mathrm{KB}$ has been identified in human pancreatic cells and this may also enhance the drive for cell survival (Wang et al 1999). EGFR signaling may also initiate early malignant transformation via activation of the Notch genes which are implicated in the regulation of cellular differentiation during pancreatic organogenesis and their activation can result in an expansion of undifferentiated cells (Miyamoto et al 2003; Heiser and Hebrok 2004).

Modulation of EGFR-mediated signaling therefore provides an attractive approach to the treatment of pancreatic cancer. The two main therapeutic modalities used to target the EGFR are monoclonal antibodies against the extracellular domain and small molecules tyrosine kinase inhibitors (TKIs) that compete at the ATP binding site of the tyrosine kinase domain (Table 1). Of the latter, erlotinib is the furthest in clinical development in pancreatic cancer.

\section{Pre-clinical evaluation of erlotinib}

Erlotinib (Tarceva; Genentech Inc, South San Francisco, CA) is an orally available quinazoline-based small molecule TKI with highly selective activity against the EGFR (invitro $\mathrm{IC}_{50}: 0.02 \mu \mathrm{mol} / \mathrm{L}$; intact cells $\left.\mathrm{IC}_{50}: 0.2 \mu \mathrm{mol} / \mathrm{L}\right)($ Moyer et al 1997; Arteaga 2001). Examples of other small molecule TKIs are provided in Table 1 and include some of the newer dual or multi-targeted TKIs with activity against more than one member of the erbB family of tyrosine kinase receptors. In pre-clinical evaluation, erlotinib demonstrated anti-tumor activity in a number of tumor xenograft models including those modeling colon, head and neck and non small cell lung cancers (Moyer et al 1997; Pollack et al 1999; Desai et al 2002). Erlotinib was shown to inhibit EGFR activation as well as to potentiate gemcitabine-induced apoptosis in pancreatic carcinoma xenografts implanted orthotopically
Table I Therapeutic approaches to targeting the epidermal growth factor receptor

\begin{tabular}{ll}
\hline Agent & Characteristics \\
\hline Cetuximab & Chimeric human-mouse IgGI Mab \\
Matuzumab (EMD 72000) & Humanised IgGI Mab \\
Panitumumab (ABX-EGF) & Fully human IgG2 Mab \\
h-R3 & Humanised IgGI Mab \\
OSI-774 (Erlotinib) & Reversible TKI \\
ZD-1839 (Gefitinib) & Reversible TKI \\
EKB-569 & Irreversible TKI \\
GW-016(Lapatinib) & EGFR/HER2, reversibleTKI \\
CI-I033 & Pan-erB TKI \\
\hline
\end{tabular}

Abbreviations: EGFR, epithelial growth factor receptor; IgG, immunogloblin; Mab, monoclonal antibody; TKI, tyrosine kinase inhibitor.

into immunodeficient mice ( $\mathrm{Ng}$ et al 2002). This enhancement of gemcitabine-induced apoptosis by erlotinib provides a reasonable scientific rationale for their combination in clinical evaluation.

Further support for the therapeutic potential of combined EGFR blockade and gemcitabine therapy has been derived from pre-clinical studies of another EGFR inhibitor, the monoclonal antibody C225 (the precursor of cetuximab); in vitro, $\mathrm{C} 225$ was shown to exert anti-proliferative effects on BxPC-3 human pancreatic cancer cells (Overholser et al 2000 ) and in an orthotopic nude mouse model of pancreatic cancer the anti-tumor effects of $\mathrm{C} 225$ were potentiated by the coadministration of gemcitabine (Bruns et al 2000). Erlotinib has been combined with a number of other cytotoxic agents in several pre-clinical models across tumor types without the demonstration of antagonism and with no clear demonstration of sequence-dependence (Akita and Sliwkowski 2003).

The mechanism of action of erlotinib in terms of the exact effects on downstream receptor signaling or activation and mechanisms of resistance to the drug are still being elucidated. Pharmacodynamic evaluation of erlotinib is crucial to this process and will be discussed later. At a preclinical level, one of the studies mentioned earlier demonstrated reduction in phosphorylation of ERK $1 / 2$ (extracellular-regulated kinase), part of the ras-Raf transduction effector cascade, in one of the xenograft models with no effect on phospho-PKB (protein kinase B), part of the PI3-kinase/Akt pathway, in either pancreatic xenograft ( $\mathrm{Ng}$ et al 2002).

Other potential cellular consequences of EGFR suppression by erlotinib include the induction of G1 cell cycle arrest possibly mediated through $\mathrm{p} 27^{\mathrm{KIP} 1}$, potentiation of apoptosis by activation of pro-apoptotic molecules such as bax and caspase- 8 , inhibition of angiogenesis through 
decreased production of growth factors such as vascular endothelial growth factor (VEGF) and inhibition of invasion and metastasis via inhibition of matrix metalloproteinases (MMPs) (Mendelsohn and Baselga 2003). There is a strong body of evidence to suggest EGFR suppression impairs the ability of cells to repair cytotoxic or radiotherapy-induced damage (Ciardiello et al 2000; Sirotnak et al 2000; Mendelsohn and Baselga 2003). This together with observations from pre-clinical studies suggests that the most effective therapeutic application of this particular targeted agent in advanced pancreatic cancer is as part of a combinatorial strategy.

Another important consideration with regards to the mode of action of EGFR inhibitors such as erlotinib is that whilst these drugs have been designed to target the EGFR, it appears that quantification of the target per se in preclinical models may not predict for responsiveness to inhibition. Erlotinib appears to inhibit both high and moderate EGFR expressing tumor cell lines suggesting that expression is not the only determinant of responsiveness to erlotinib (Desai et al 2002). Although variability does exist in EGFR quantification methodology, it is likely that other factors such as receptor mutation, gene amplification, dimerisation partners (such as Her2), receptor cross-talk and alternative pathways for downstream activation may be confounding factors. Taking these into account quantification of EGFR status may not be reflective of dependence of cell survival/proliferation on EGFR signaling which may be a determinant of response to EGFR inhibitors.

\section{Early phase studies: pharmacology and pharmacokinetics}

Four initial phase I trials, two of which were conducted in volunteers, established preliminary pharmacokinetic (PK) data for erlotinib (Hidalgo et al 2001; Hidalgo and Bloedow 2003). These demonstrated rapid absorption with peak plasma concentrations occurring within 4 hours, dose dependent PK between $3 \mathrm{mg}$ to $30 \mathrm{mg}$ but non-linear PK at higher doses, drug accumulation and continuous exposure with daily treatment (Hidalgo et al 2001; Hidalgo and Bloedow 2003). The phase I study that established the current recommended dosing for erlotinib was performed in patients with advanced, refractory solid tumors and explored a daily dosing of dose-escalated erlotinib according to three different schedules (Hidalgo et al 2001). A daily dosing schedule was established as tolerable with PK indicating increased plasma levels of erlotinib with escalating doses and no unexpected drug accumulation.
Erlotinib appeared to be well tolerated and diarrhea and acneiform skin rash were defined as the dose-limiting toxicities, dose-limiting diarrhea occurring at the $200 \mathrm{mg} /$ day dose. At a dose of $150 \mathrm{mg} /$ day diarrhea appeared to be manageable as was acneiform rash which was seen to occur at all doses of erlotinib. This dose was subsequently recommended as the dose to be taken forward in further clinical evaluation.

On a continuous dosing schedule, the steady state was reached in seven days with a half life of approximately 18 hours (Hidalgo et al 2001). Further support for the $150 \mathrm{mg} /$ day dosing was provided by the fact that the minimum plasma concentration at this dose exceeded $500 \mathrm{mg} / \mathrm{mL}$, which is the concentration associated with EGFR inhibition and anti-tumor activity in pre-clinical studies (Hidalgo et al 2001). Plasma concentrations in this range were seen less frequently with dosing of $50 \mathrm{mg}$ or $100 \mathrm{mg}$ per day.

Erlotinib undergoes hepatic metabolism, $80 \%$ occurring via cytochrome CYP3A4 which is commonly involved in the hepatic metabolism of several other drugs thereby increasing the potential for drug-drug interaction (Hidalgo and Bloedow 2003). In particular potent 3A4 inhibitors such as ketoconazole, certain other anti-fungals (fluconazole and itraconazole) and antibiotics (ie, erythromycin) may increase exposure to erlotinib whilst potent $3 \mathrm{~A} 4$ inducers such as rifampicin and some anti-epileptic drugs will reduce exposure (Abbas et al 2003). Caution should be exercised when co-administering these drugs with erlotinib, particularly the CA4 inhibitors, and dose adjustment of the erlotinib may be necessary. The predominant metabolite of erlotinib is OSI-420 which has a similar pharmacokinetic profile to erlotinib, also inhibits EGFR and undergoes biliary excretion (Moyer et al 1997).

Combination of cytotoxics with other agents such as erlotinib is intuitively attractive in terms of the potential for targeting different oncogenic processes, avoiding development of resistance, potential for synergy and the resultant possible enhancement of anti-tumor effect therein. The pre-clinical rationale for combining gemcitabine with erlotinib has already been stated. In phase I evaluation in patients with pancreatic cancer, the combination of gemcitabine administered according to the standard schedule plus erlotinib at doses of $100 \mathrm{mg} /$ day or $150 \mathrm{mg}$ /day was found to be tolerable with no dose-limiting toxicity or significant pharmacokinetic interactions (Dragovich et al 2003; Porterfield et al 2004). In another phase I study, the combination of erlotinib with the chemotherapy doublet gemcitabine (according to a standard schedule) and cisplatin 
in patients with advanced solid tumors was tolerable and did not indicate significant pharmacokinetic interactions (Ratain et al 2002). These studies provide support for evaluation of a gemcitabine-based chemotherapy plus erlotinib in the treatment of pancreatic cancer.

\section{Erlotinib in combination with chemotherapy in clinical studies}

Initially erlotinib was evaluated as monotherapy in phase II studies across a broad range of tumor types including lung, ovary, colorectal, and head and neck cancer (Herbst 2003; Shepherd et al 2004; Soulieres et al 2004) with the demonstration of responses and induction of stable disease in several of these studies. Notably, the demonstration of single agent activity for erlotinib in the treatment of chemorefractory lung cancer led to regulatory approval for this indication. Toxicities were in line with those seen in earlier phase I evaluation.

In pancreatic cancer, clinical evaluation of erlotinib has been undertaken as part of a combination approach with gemcitabine. The National Canadian Institute of Cancer (NCIC) conducted an international, multicenter randomized placebo controlled phase III trial (PA.3) of gemcitabine versus gemcitabine in combination with erlotinib in advanced pancreatic cancer (Moore et al 2005). 569 patients were randomized to receive either gemcitabine, which was administered at a dose of $1000 \mathrm{mg} / \mathrm{m}^{2}$ over 30 minutes given weekly for seven weeks then for three out of every four weeks thereafter or gemcitabine (same schedule) plus erlotinib. The starting dose of erlotinib in the combination arm was $100 \mathrm{mg} /$ day based on the potential for additive toxicity. After several planned safety reviews, including a review of toxicity by the Drug Safety Monitoring Committee, a smaller final cohort of patients received the higher dose of $150 \mathrm{mg}$ daily. Expression of EGFR was not mandated for trial entry although this information was collected. Patient characteristics were well balanced and approximately $25 \%$ of patients in both arms had locally advanced disease.

Overall survival was significantly better in the erlotinib arm compared with the placebo controlled arm with a median survival of 6.37 versus 5.91 months (HR 0.81; 95\% CI 0.67, $0.97 ; \mathrm{p}=0.025$ ) and one year survival of $24 \%$ versus $17 \%$ respectively. Whilst the difference in median survival appears small, it should be noted that the survival curves came closer together for this point estimate and that the HR is probably more representative of the overall difference between the two arms. Similarly, progression-free survival was significantly improved in the combination arm, the medians being 3.75 versus 3.55 months respectively $(\mathrm{HR}=0.76 ; 95 \%$ CI $0.63,0.91 ; \mathrm{p}=0.003)$. Overall response rate did not appear to be different between the combination and placebo controlled arms (8.6\% vs $8.0 \%$ respectively) although disease control did appear different (57.5\% vs 49.2\%). Toxicity will be discussed later but skin rash and diarrhea appeared to be increased in the erlotinib arm. Quality of Life assessment was undertaken in the North American centers according to the EORTC QLQ-c30 and indicated that per cycle there was a significant difference in favor of the placebo controlled arm in terms of diarrhea. There were no significant differences for global quality of life scores (Moore et al 2005). Although the survival increment in this study was small, it is now one of two positive randomized studies demonstrating a survival benefit for combining gemcitabine with another agent, importantly a biologic response modifier. The study also led to regulatory approval for the combination of gemcitabine and erlotinib in advanced pancreatic cancer in the US in November 2005. Given that the majority of patients had been treated with the $100 \mathrm{mg}$ dose in the trial, this has been recommended as the combination dose.

These results are in contrast to those observed in two randomized studies of chemotherapy doublets plus or minus erlotinib in over 1000 patients each (TRIBUTE and TALENT) performed in chemonaive lung cancer patients (Gatzemeier et al 2004; Herbst, Johnson, et al 2005). Importantly, presence of EGFR mutations in the tyrosine kinase domain and other molecular aberrations in lung cancer patients are associated with response/resistance to EGFR TKIs (Lynch et al 2004; Eberhard et al 2005) and therefore the lack of selection for potential responders may have diluted any therapeutic effect of erlotinib in these large studies. EGFR mutations have not been found in pancreatic cancers and this is therefore unlikely to be a molecular marker that could potentially facilitate better selection of patients for erlotinib therapy. This will be discussed in more detail in later.

Combination of erlotinib with an effective cytotoxic doublet such as gemcitabine plus capecitabine would appear to be an attractive therapeutic option. Furthermore combination of erlotinib with another biologic agent such as the anti-VEGF agent bevacizumab, also appears attractive. In colon and gastric cancer cell lines, combined blockade of EGFR and VEGF resulted in significant antitumor responses which were greater than suppression of one pathway alone (Ciardiello et al 2000; Jung et al 2002). 
In a phase I/II study in lung cancer, the combination of erlotinib and bevacizumab was tolerable, had no pharmacokinetic interaction and importantly resulted in responses in the absence of cytotoxic treatment (Herbst, Prager, et al 2005). Currently, there is an international placebo controlled phase III study underway comparing the combination of gemcitabine plus erlotinib according to the PA.3 schedule versus gemcitabine, erlotinib plus bevacizumab in patients with metastatic pancreatic cancer (Figure 2), the results of which should prove very interesting. The erlotinib dose being used is $100 \mathrm{mg}$ /day and the study is powered to demonstrate a survival difference between the arms.

At the Royal Marsden Hospital, UK, a phase I/II study (TARGET) is exploring the combination of gemcitabine, capecitabine (according to the GEMCAP schedule) plus erlotinib and bevacizumab in locally advanced and metastatic pancreatic cancer. It is likely that these multiagent, multi-targeted approaches will be increasingly investigated in the treatment of advanced pancreatic cancer. In addition studies of alternative EGFR inhibitors in the first-line treatment of advanced pancreatic cancer are in progress with the randomised phase III Southwest Oncology Group (SWOG) trial of gemcitabine versus gemcitabine plus cetuximab having recently completed accrual. In a randomized phase II study performed by the University of Chicago, patients with advanced pancreatic cancer are randomized between gemcitabine, bevacizumab and erlotinib or gemcitabine, bevacizumab and cetuximab which will facilitate selection of a future comparator arm for phase III evaluation.

\section{Toxicities associated with erlotinib Skin rash}

A class effect of EGFR inhibitors such as erlotinib and indeed the monoclonal antibodies is the development of a skin rash characterized by clusters of monomorphic pustular lesions and which is often likened to an acneiform eruption. The distribution of the rash is usually limited to the face and upper torso and although grading in the Common Toxicity Criteria version 2.0 categorizes rash according to percentage of body distribution, the latest version, 3.0,

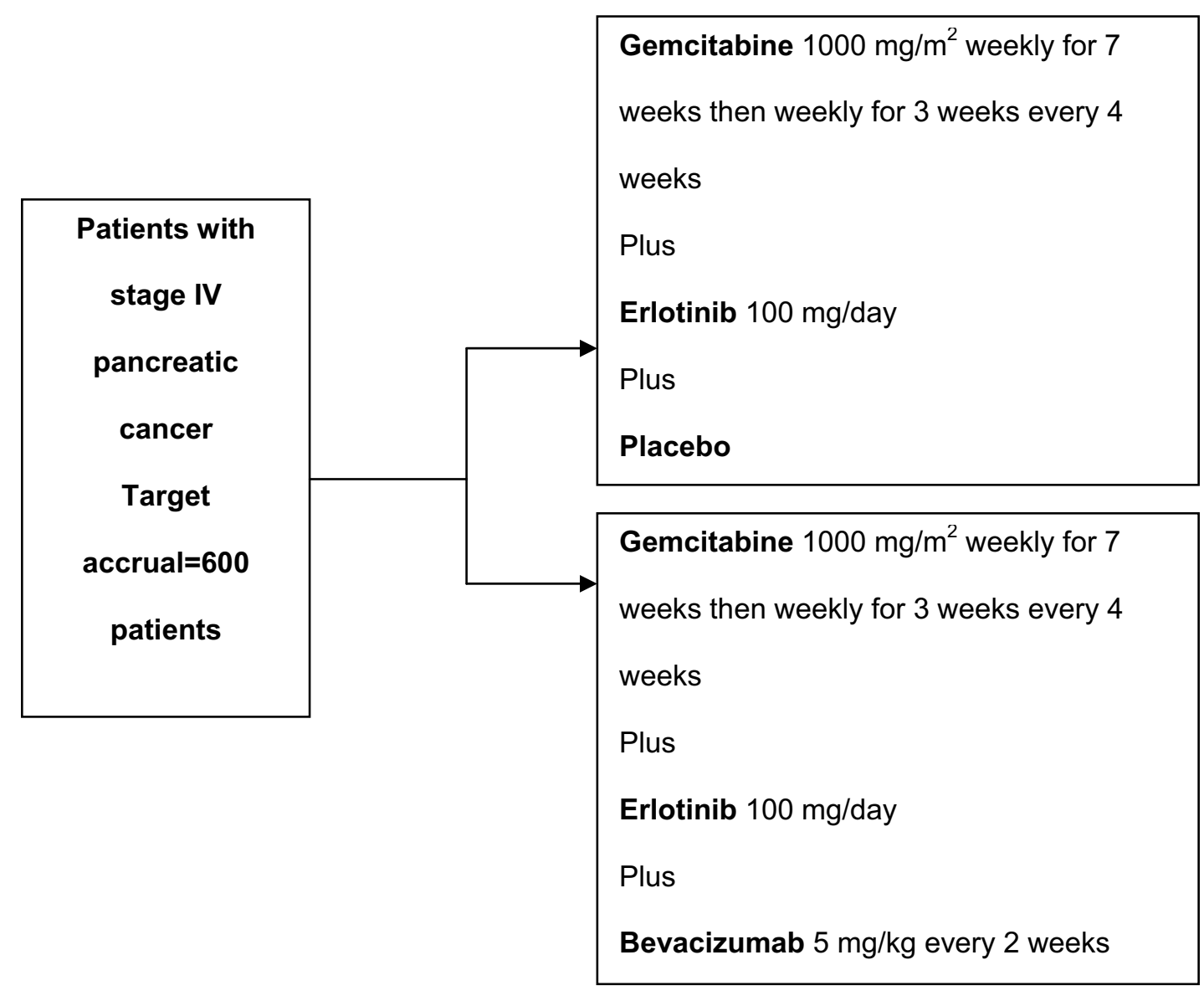

Figure 2 Study schema for the AVITA randomized Phase III study. 
perhaps more appropriately allows grading of acneiform rash according to the need for medical intervention, although even the latest classification is not ideal.

Other dermatological manifestations of EGFR inhibition include dry skin, pruritis, erythema and nail, hair and eyelash changes (Perez-Soler and Saltz 2005). The onset of rash is usually within the first two weeks of therapy and often improves despite further continuous dosing (Hidalgo et al 2001). Erlotinib-induced rash also appears to be dosedependent (Hidalgo et al 2001) in line with the dosedependence demonstrated for another EGFR TKI in clinical use, gefitinib (Perez-Soler and Saltz 2005). The rash is usually well tolerated but when associated with symptoms such as itching or supra-added infection can be very problematic. Furthermore, the cosmetic appearance of the rash can be unacceptable to the patient and impact on their quality of life.

In the initial phase I studies biopsy of affected areas revealed neutrophil infiltration of dermal tissues, particularly in relation to the infundibular portion of the hair follicle, and thinning of the stratum corneum layer of the epidermis (Hidalgo et al 2001; Malik et al 2003). The etiology of this skin reaction is not entirely clear but may relate to disruption of EGFR signaling in the dermis. EGFR is expressed in normal keratinocytes, skin fibroblasts, and in the outer route sheath of the hair follicle and is likely to have a physiological role in maintenance of the epidermis and skin/hair development (King et al 1990; Hansen et al 1997). Alternatively, EGFR inhibition may induce a local or systemic inflammatory skin reaction (Perez-Soler and Saltz 2005).

Pharmacogenomics may play a role in the inter-patient variability of rash development with a recent study suggesting the number of germline polymorphic CA dinucleotide repeats in intron 1 of the EGFR gene may be associated with response to EGFR inhibition and rash; cell lines (mainly head and neck) demonstrating fewer than 36 CA repeats were more sensitive to inhibition by erlotinib than those with greater than or equal to $36 \mathrm{CA}$ repeats (Amador et al 2004). In the same study, a smaller number of CA repeats appeared to correlate with development of skin rash in patients with colorectal cancer $(n=19)$ treated with gefitinib. These results are of interest and importantly indicate a potential mechanism for the association of rash with outcome which will be discussed further, but do require validation in larger studies.

The therapeutic management of EGFR inhibitor-induced skin rash has generated significant debate and there are currently no evidence-based guidelines for treatment. A variety of dermatological interventions have been employed including topical and systemic antibiotics, retinoids, corticosteroids and emollients with varying degrees of success (Perez-Soler and Saltz 2005; Segaert and Van Cutsem 2005). Certainly where there is clear evidence of supra-added infection, antibiotics may be beneficial. Otherwise, experience with all these agents is largely anecdotal and there is a clear need for systematic evaluation of interventions within controlled trials.

\section{Other toxicities}

Other commonly observed toxicities in the phase I studies and the Canadian phase III study were diarrhea, headache, nausea, vomiting, fatigue and mucositis (Hidalgo et al 2001; Dragovich et al 2003; Porterfield et al 2004; Moore et al 2005). Table 2 demonstrates the toxicities seen in the NCIC study. Apart from skin rash, the rate of grade 3/4 diarrhea was different between the arms being $6 \%$ in the gemcitabine/ erlotinib arm and $2 \%$ in the gemcitabine/placebo arm. Erlotinib-induced diarrhea should be managed aggressively with medical assessment, admission and the use of antidiarrheal agents and parenteral fluids as necessary. Both gemcitabine and erlotinib can cause interstitial fibrosis (pneumonitis) and in the NCIC study this occurred in $2 \%$ of patients receiving combination therapy compared with $<1 \%$ who received gemcitabine alone. The overall toxicity profile of erlotinib in combination with gemcitabine, however, appears to be tolerable.

\section{Selection of patients for therapy}

The issue of patient selection with targeted agents is important in terms of optimizing the chance of response to therapy, limiting exposure to drugs that do add additional toxicity and to help rationalize resources. As discussed earlier, skin rash may be a surrogate marker of outcome to EGFR inhibitors such as erlotinib. Although this association has been less consistent with other EGFR TKIs such as gefitinib (Perez-Soler and Saltz 2005) it appears to be stronger with erlotinib as demonstrated in a number of trials, predominantly in lung cancer (Gatzmeier et al 2004; Herbst, Johnson, et al 2005; Perez-Soler and Saltz 2005). Not only the presence but the intensity of skin rash appeared to correlate with survival in these trials. Certainly in the NCIC Phase III study in pancreatic cancer, sub-group analysis also suggested an association with skin rash and survival; median survival for patients with grade 0 skin rash $(n=79)$ was 5.29 months, with grade 1 skin rash $(n=108)$ was 5.75 months and with grade 3 skin rash $(n=103)$ was 10.51 months 
Table 2 Toxicities associated with the combination of erlotinib and gemcitabine in the NCIC Phase III study

\begin{tabular}{|c|c|c|c|c|}
\hline \multirow[t]{2}{*}{ Toxicity } & \multicolumn{2}{|c|}{ Gemcitabine plus erlotinib (\%) $n=282$} & \multicolumn{2}{|c|}{ Gemcitabine plus placebo (\%) $n=280$} \\
\hline & Any & Grade $3 / 4$ & Any & Grade $3 / 4$ \\
\hline Rash & 72 & 6 & 29 & I \\
\hline Diarrhea & 56 & 6 & 41 & 2 \\
\hline Neutropaenia & 31 & 25 & 28 & 26 \\
\hline Thrombocytopaenia & 64 & 10 & 66 & 12 \\
\hline Infection & 43 & 17 & 34 & 16 \\
\hline Stomatitis & 23 & $<1$ & 14 & 0 \\
\hline Pneumonitis & 2 & 2 & 1 & $<1$ \\
\hline Fatigue & 89 & 15 & 86 & 15 \\
\hline Dehydration & 8 & 3 & 10 & 5 \\
\hline
\end{tabular}

Abbreviations: $\mathrm{NCIC}$, National Cancer Institute of Canada.

(Moore et al 2005). The difference in survival between patients who developed grade 2 rash compared with grade $0 / 1$ was statistically significant $(p<0.0001)$. However, titration of erlotinib to skin rash in an attempt to optimize efficacy is likely to be difficult given that diarrhea has been demonstrated as the main dose-limiting toxicity. A dose-torash study is currently ongoing with erlotinib in lung cancer to explore this issue further (Mita et al 2005).

Several pharmacodynamic studies have been undertaken, often using skin as a surrogate tissue for response, in order to identify molecular aberrations in the EGFR pathway that may indicate response or resistance to therapy (Albanell et al 2002; LoRusso 2003; Malik et al 2003). However, whilst these often reveal interesting hypothesis-generating observations, none has identified a marker that has immediate clinical utility (Abanell et al 2002; Malik et al 2003). Furthermore, molecular changes occurring in response to EGFR suppression in the skin may not mirror changes in the tumor for a number of reasons including differences in EGFR dimerization partners and the presence of somatic mutations (Baselga 2003; Laux et al 2006).

Across studies and tumor types, there has consistently been a lack of association between EGFR expression, as judged by immunohistochemistry, and response to therapy in line with the pre-clinical observations (Desai et al 2002; Herbst 2003; Hortobagyi and Sauter 2003). In the NCIC study in pancreatic cancer, EGFR expression did not correlate with survival by treatment arm with the cut-off for EGFR positive tumors being $10 \%$ membranous staining (Moore et al 2005). Selection for erlotinib therapy in pancreatic cancer should not be made on the basis of EGFR expression by immunohistochemistry. At the present time, there are no validated predictive markers that allow rationalization of erlotinib use in pancreatic cancer but work in this area is ongoing.

\section{Conclusion}

Erlotinb in combination with gemcitabine has recently been shown to be superior to gemcitabine monotherapy with a very modest improvement in survival. There are now two positive randomized phase III trials which have demonstrated a survival advantage for gemcitabine-based combination therapy, one incorporating a cytotoxic agent (Cunningham et al 2005) and the other, a biologic agent directed against the EGFR pathway (Moore et al 2005). Both represent important, albeit small, steps in improving outcome from this aggressive malignancy and the latter importantly validating an approach that has been rationally selected based on the perceived importance of one of the pathophysiological mechanisms underlying the disease. Both could be considered standard treatment options for advanced pancreatic cancer. However, quality of life and pharmacoeconomic considerations are likely to dictate the uptake of either approach and the latter are yet to be undertaken. Ongoing and future studies in advanced pancreatic cancer are building on combinatorial approaches and the next generation of trials are incorporating biologic agents with conventional cytotoxics and additionally combining biologic agents. This multi-targeted approach may ultimately prove more effective in treating what is effectively a chemo-resistant disease. Given the efficacy of erlotinib demonstrated in the Canadian trial, erlotinib or alternative EGFR inhibitors such as cetuximab, are likely to be part of this strategy.

\section{References}

Abbas R, Fettner S, Riek M, et al. 2003. A drug-drug interaction study to evaluate the effect of rifampicin on the pharmacokinetics of the EGF receptor tyrosine kinase inhibitor, erlotinib, in healthy subjects [abstract]. Proc Am Soc Clin Oncol, 22:548.

Akita RW, Sliwkowski MX. 2003. Preclinical studies with erlotinib (Tarceva). Semin Oncol, 30(3 Suppl 7):15-24. 
Albanell J, Rojo F, Averbuch S, et al. 2002. Pharmacodynamic studies of the epidermal growth factor receptor inhibitor ZD1839 in skin from cancer patients: histopathologic and molecular consequences of receptor inhibition. $J$ Clin Oncol, 20:110-24.

Amador ML, Oppenheimer D, Perea S, et al. 2004. An epidermal growth factor receptor intron 1 polymorphism mediates response to epidermal growth factor receptor inhibitors. Cancer Res, 64:913943.

Arteaga CL. 2001. The epidermal growth factor receptor: from mutant oncogene in nonhuman cancers to therapeutic target in human neoplasia. J Clin Oncol, 19(18 Suppl):32S-40S.

Arteaga C. 2003. Targeting HER1/EGFR: a molecular approach to cancer therapy. Semin Oncol, 30(3 Suppl 7):3-14.

Baselga J. 2003. Skin as a surrogate tissue for pharmacodynamic end points: is it deep enough? Clin Cancer Res, 9:2389-90.

Bondar VM, Sweeney-Gotsch B, Andreeff M, et al. 2002. Inhibition of the phosphatidylinositol 3'-kinase-AKT pathway induces apoptosis in pancreatic carcinoma cells in vitro and in vivo. Mol Cancer Ther, 1:989-97.

Bramhall SR, Rosemurgy A, Brown PD, et al. 2001. Marimastat as firstline therapy for patients with unresectable pancreatic cancer: a randomized trial. J Clin Oncol, 19:3447-55.

Bramhall SR, Schulz J, Nemunaitis J, et al. 2002. A double-blind placebocontrolled, randomised study comparing gemcitabine and marimastat with gemcitabine and placebo as first line therapy in patients with advanced pancreatic cancer. Br J Cancer, 87:161-7.

Bruns CJ, Harbison MT, Davis DW, et al. 2000. Epidermal growth factor receptor blockade with $\mathrm{C} 225$ plus gemcitabine results in regression of human pancreatic carcinoma growing orthotopically in nude mice by antiangiogenic mechanisms. Clin Cancer Res, 6:1936-48.

Burris HA III, Moore MJ, Andersen J, et al. 1997. Improvements in survival and clinical benefit with gemcitabine as first-line therapy for patients with advanced pancreas cancer: a randomized trial. J Clin Oncol, 15:2403-13.

Cheverton P, Friess H, Andras C, et al. 2004. Phase III results of exatecan (DX-8951f) versus gemcitabine in chemotherapy-naive patients with advanced pancreatic cancer [abstract]. Proc Am Soc Clin Oncol, 23:4005

Ciardiello F, Bianco R, Damiano V, et al. 2000. Antiangiogenic and antitumor activity of anti-epidermal growth factor receptor $\mathrm{C} 225$ monoclonal antibody in combination with vascular endothelial growth factor antisense oligonucleotide in human GEO colon cancer cells. Clin Cancer Res, 6:3739-47.

Ciardiello F, Caputo R, Bianco R, et al. 2000. Antitumor effect and potentiation of cytotoxic drugs activity in human cancer cells by ZD1839 (Iressa), an epidermal growth factor receptor-selective tyrosine kinase inhibitor. Clin Cancer Res, 6:2053-63.

Cunningham D, Chau I, Stocken D, et al. 2005. Phase III randomised comparison of gemcitabine versus gemcitabine plus capecitabine in patients with advanced pancreatic cancer. Eur J Cancer, 3:12.

Desai B, Higgins B, Smith M. 2002. Antitumour activity of the EGFR/TK inhibitor Tarceva (erlotinib, OSI-774) tumour models [abstract]. Eur $J$ Cancer, 38:203.

Dragovich T, Patnaik A, Rowinsky EK, et al. 2003. A phase I B trial of gemcitabine and erlotinib HCL in patients with advanced pancreatic adenocarcinoma and other potentially responsive malignancies [abstract]. Proc Am Soc Clin Oncol, 22:223.

Eberhard DA, Johnson BE, Amler LC, et al. 2005. Mutations in the epidermal growth factor receptor and in KRAS are predictive and prognostic indicators in patients with non-small-cell lung cancer treated with chemotherapy alone and in combination with erlotinib. $J$ Clin Oncol, 23:5900-9.

Ebert MP, Fei G, Schandl L, et al. 2002. Reduced PTEN expression in the pancreas overexpressing transforming growth factor-beta $1 . \mathrm{Br} J$ Cancer, 86:257-62.
Gatzemeier U, Pluzanska A, Szczesna A, et al. 2004. Results of a phase III trial of erlotinib (OSI-774) combined with cisplatin and gemcitabine (GC) chemotherapy in advanced non-small cell lung cancer (NSCLC) [abstract]. J Clin Oncol, 22(14S)7010.

Guttridge DC, Albanese C, Reuther JY, et al. 1999. NF-KappaB controls cell growth and differentiation through transcriptional regulation of cyclin D1. Mol Cell Biol, 19:5785-99.

Habib AA, Chatterjee S, Park SK, et al. 2001. The epidermal growth factor receptor engages receptor interacting protein and nuclear factor-kappa B (NF-Kappa B)-inducing kinase to activate NF-kappa B. Identification of a novel receptor-tyrosine kinase signalosome. $J$ Biol Chem, 276:8865-74.

Hansen LA, Alexander N, Hogan ME, et al. 1997. Genetically null mice reveal a central role for epidermal growth factor receptor in the differentiation of the hair follicle and normal hair development. Am J Pathol, 150:1959-75.

Heiser PW, Hebrok M. 2004. Development and cancer: lessons learned in the pancreas. Cell Cycle, 3:270-2.

Herbst RS. 2003. Erlotinib (Tarceva): an update on the clinical trial program. Semin Oncol, 30(3 Suppl 7):34-46.

Herbst RS, Johnson DH, Mininberg E, et al. 2005. Phase I/II trial evaluating the anti-vascular endothelial growth factor monoclonal antibody bevacizumab in combination with the HER-1/epidermal growth factor receptor tyrosine kinase inhibitor erlotinib for patients with recurrent non-small-cell lung cancer. $J$ Clin Oncol, 23:2544-55.

Herbst RS, Prager D, Hermann R, et al. 2005. TRIBUTE: a Phase III trial of erlotinib hydrochloride (OSI-774) combined with carboplatin and paclitaxel chemotherapy in advanced non-small-cell lung cancer. $J$ Clin Oncol, 23:5892-9.

Herrmann R, Bodoky G, Ruhstaller T, et al. 2005. Gemcitabine (G) plus Capecitabine (C) versus $\mathrm{G}$ alone in locally advanced or metastatic pancreatic cancer. A randomized phase III study of the Swiss Group for Clinical Cancer Research (SAKK) and the Central European Cooperative Oncology Group (CECOG) [abstract]. J Clin Oncol, 23(16S):4010.

Hidalgo M, Bloedow D. 2003. Pharmacokinetics and pharmacodynamics: maximizing the clinical potential of erlotinib (Tarceva). Semin Oncol, 30(3 Suppl 7):25-33.

Hidalgo M, Siu LL, Nemunaitis J, et al. 2001. Phase I and pharmacologic study of OSI-774, an epidermal growth factor receptor tyrosine kinase inhibitor, in patients with advanced solid malignancies. J Clin Oncol, 19:3267-79.

Hinz M, Krappmann D, Eichten A, et al. 1999. NF-kappaB function in growth control: regulation of cyclin D1 expression and G0/G1-to-Sphase transition. Mol Cell Biol, 19:2690-8.

Hortobagyi GN, Sauter G. 2003. Challenges and opportunities for erlotinib (Tarceva): what does the future hold? Semin Oncol, 30(3 Suppl 7):4753.

Jung YD, Mansfield PF, Akagi M, et al. 2002. Effects of combination anti-vascular endothelial growth factor receptor and anti-epidermal growth factor receptor therapies on the growth of gastric cancer in a nude mouse model. Eur J Cancer, 38:1133-40.

King LE Jr, Gates RE, Stoscheck CM, et al. 1990. The EGF/TGF alpha receptor in skin. J Invest Dermatol, 94(6 Suppl):164S-70S.

Laux I, Jain A, Singh S, et al. 2006. Epidermal growth factor receptor dimerization status determines skin toxicity to HER-kinase targeted therapies. Br J Cancer, 94:85-92.

Lemoine NR, Hughes CM, Barton CM, et al. 1992. The epidermal growth factor receptor in human pancreatic cancer. $J$ Pathol, 166:7-12.

LoRusso PM. 2003. Phase I Studies of ZD1839 in patients with common solid tumors. Semin Oncol, 30(1 Suppl 1):21-9.

Louvet C, Labianca R, Hammel P, et al. 2005. Gemcitabine in combination with oxaliplatin compared with gemcitabine alone in locally advanced or metastatic pancreatic cancer: results of a GERCOR and GISCAD Phase III Trial. J Clin Oncol, 23:3509-16. 
Lynch TJ, Bell DW, Sordella R, et al. 2004. Activating mutations in the epidermal growth factor receptor underlying responsiveness of nonsmall-cell lung cancer to gefitinib. $N$ Engl J Med, 350:2129-39.

Malik SN, Siu LL, Rowinsky EK, et al. 2003. Pharmacodynamic evaluation of the epidermal growth factor receptor inhibitor OSI-774 in human epidermis of cancer patients. Clin Cancer Res, 9:2478-86.

Marmor MD, Skaria KB, Yarden Y. 2004. Signal transduction and oncogenesis by ErbB/HER Receptors. Int J Radiat Oncol Biol Phys, 58:903-13.

Mendelsohn J, Baselga J. 2003. Status of epidermal growth factor receptor antagonists in the biology and treatment of cancer. $J$ Clin Oncol, 21:2787-99.

Mita CA, Schwartz G, Mita MM, et al. 2005. A pilot, pharmacokinetic (PK), and pharmacodynamic (PD) study to determine the feasibility of intrapatient dose escalation to tolerable rash and the activity of maximal doses of erlotinib (E) in previously treated patients with advanced non-small cell lung cancer (NSCLC) [abstract]. J Clin Oncol, 23(16S):3045

Miyamoto Y, Maitra A, Ghosh B, et al. 2003. Notch mediates TGF alphainduced changes in epithelial differentiation during pancreatic tumorigenesis. Cancer Cell, 3:565-76.

Moore MJ, Hamm J, Dancey J, et al. 2003. Comparison of gemcitabine versus the matrix metalloproteinase inhibitor BAY 12-9566 in patients with advanced or metastatic adenocarcinoma of the pancreas: a Phase III Trial of the National Cancer Institute of Canada Clinical Trials Group. J Clin Oncol, 21:3296-302.

Moore MJ, Goldstein D, Hamm J, et al. 2005. Erlotinib plus gemcitabine compared to gemcitabine alone in patients with advanced pancreatic cancer. A phase III trial of the National Cancer Institute of Canada Clinical Trials Group (NCIC-CTG) [abstract]. J Clin Oncol, 23(16S): 1

Moyer JD, Barbacci EG, Iwata KK, et al. 1997. Induction of apoptosis and cell cycle arrest by CP-358,774, an inhibitor of epidermal growth factor receptor tyrosine kinase. Cancer Res, 57:4838-48.

$\mathrm{Ng}$ SS, Tsao MS, Nicklee T, et al. 2002. Effects of the epidermal growth factor receptor inhibitor OSI-774, tarceva, on downstream signaling pathways and apoptosis in human pancreatic adenocarcinoma. Mol Cancer Ther, 1:777-83.

Ogiso H, Ishitani R, Nureki O, et al. 2002. Crystal structure of the complex of human epidermal growth factor and receptor extracellular domains. Cell, 110:775-87.

Overholser JP, Prewett MC, Hooper AT, et al. 2000. Epidermal growth factor receptor blockade by antibody IMC-C225 inhibits growth of a human pancreatic carcinoma xenograft in nude mice. Cancer, 89:74-82.

Perez-Soler R, Saltz L. 2005. Cutaneous adverse effects With HER1/EGFRtargeted agents: is there a silver lining? J Clin Oncol, 23:5235-46.

Perren A, Komminoth P, Saremaslani P, et al. 2000. Mutation and expression analyses reveal differential subcellular compartmentalization of PTEN in endocrine pancreatic tumors compared to normal islet cells. Am J Pathol, 157:1097-103.

Pollack VA, Savage DM, Baker DA, et al. 1999. Inhibition of epidermal growth factor receptor-associated tyrosine phosphorylation in human carcinomas with CP-358,774: dynamics of receptor inhibition in situ and antitumor effects in athymic mice. J Pharmacol Exp Ther, 291:739-48.
Porterfield BW, Dragovich T, Patnaik A, et al. 2004. Erlotinib + gemcitabine in patients with unresectable pancreatic carcinoma: Results from a phase IB trial [abstract]. J Clin Oncol, 22(14S):4110.

Ratain MJ, George CM, Janisch L, et al. 2002. Phase I trial of erlotinib (OSI-774) in combination with gemcitabine $(\mathrm{G})$ and cisplatin $(\mathrm{P})$ in patients with advanced solid tumors [abstract]. Proc Am Soc Clin Oncol, 21:2115

Richards DA, Kindler HL, Oettle H, et al. 2004. A randomized phase III study comparing gemcitabine plus pemetrexed versus gemcitabine in patients with locally advanced or metastatic pancreatic cancer [abstract]. Proc Am Soc Clin Oncol, 23:4007.

Rocha Lima CM, Green MR, Rotche R, et al. 2004. Irinotecan plus gemcitabine results in no survival advantage compared with gemcitabine monotherapy in patients with locally advanced or metastatic pancreatic cancer despite increased tumor response rate. Clin Oncol, 22:3776-83.

Ruggeri BA, Huang L, Wood M, et al. 1998. Amplification and overexpression of the AKT2 oncogene in a subset of human pancreatic ductal adenocarcinomas. Mol Carcinog, 21:81-6.

Schlieman MG, Fahy BN, Ramsamooj R, et al. 2003. Incidence, mechanism and prognostic value of activated AKT in pancreas cancer. Br JCancer, 89:2110-15.

Segaert S, Van Cutsem E. 2005. Clinical signs, pathophysiology and management of skin toxicity during therapy with epidermal growth factor receptor inhibitors. Ann Oncol, 16:1425-33.

Shepherd FA, Pereira J, Ciuleanu TE, et al. 2004. A randomized placebo-controlled trial of erlotinib in patients with advanced nonsmall cell lung cancer (NSCLC) following failure of 1 st line or 2nd line chemotherapy. A National Cancer Institute of Canada Clinical Trials Group (NCIC-CTG) trial [abstract]. J Clin Oncol, 22(14S):7022

Sirotnak FM, Zakowski MF, Miller VA, et al. 2000. Efficacy of cytotoxic agents against human tumor xenografts is markedly enhanced by coadministration of ZD1839 (Iressa), an inhibitor of EGFR tyrosine kinase. Clin Cancer Res, 6:4885-92.

Soulieres D, Senzer NN, Vokes EE, et al. 2004. Multicenter Phase II study of erlotinib, an oral epidermal growth factor receptor tyrosine kinase inhibitor, in patients with recurrent or metastatic squamous cell cancer of the head and neck. J Clin Oncol, 22:77-85.

Tempero M, Plunkett W, Ruiz Van Haperen V, et al. 2003. Randomized Phase II comparison of dose-intense gemcitabine: thirty-minute infusion and fixed dose rate infusion in patients with pancreatic adenocarcinoma. J Clin Oncol, 21:3402-8.

Van Cutsem E, van de Velde H, Karasek P, et al. 2004. Phase III trial of gemcitabine plus tipifarnib compared with gemcitabine plus placebo in advanced pancreatic cancer. J Clin Oncol, 22:1430-8.

Wang W, Abbruzzese JL, Evans DB, et al. 1999. The nuclear factor-kappa B RelA transcription factor is constitutively activated in human pancreatic adenocarcinoma cells. Clin Cancer Res, 5:119-27.

Yamanaka Y, Friess H, Kobrin MS, 1993. Overexpression of HER2/neu oncogene in human pancreatic carcinoma. Hum Pathol, 24:1127-34.

Yarden Y, Sliwkowski MX. 2001. Untangling the ErbB signalling network. Nat Rev Mol Cell Biol, 2:127-37. 
